\title{
Photoexpansion of Biobased Polyesters: Mechanism Analysis by Time-Resolved Measurements of an Amorphous Polycinnamate Hard Film
}

Kenji Takada, ${ }^{a}$ Katsuaki Yasaki, ${ }^{a}$ Sakshi Rawat,${ }^{a}$ Kosuke Okeyoshi, ${ }^{a}$ Amit Kumar, ${ }^{a, b}$ Hideyuki Murata $^{a}$ and Tatsuo Kaneko ${ }^{a}$

${ }^{a}$ Graduate School of Advanced Science and Technology, Japan Advanced Institute of Science and Technology, 1-1 Asahidai, Nomi, Ishikawa, 923 1292, Japan,

${ }^{\mathrm{b}}$ University of Delhi, Benito Juarez Marg, South Campus, South Moti Bagh, New Delhi, Delhi 110021, India.

* Corresponding Author, E-mail: kaneko@ jaist.ac.jp 


\section{Contents}

${ }^{1} \mathrm{H}$ NMR spectrum of the P3HCA and PdHCA (Figure S1) 3

IR spectra of the P3HCA and PdHCA (Figure S2 and S3) 4

UV-vis spectra of P3HCA and PdHCA films (Figure S4) 5

IR/ATR spectra of cross-section of P3HCA film (Figure S5)

For determine the changes of the vinyl bond of polymers from IR spectrum (Figure S6) 6

$\begin{array}{ll}\text { Schematic illustration of structural changes for PdHCA (Figure S7) } & 7\end{array}$

Different deformation pattern of the P3HCA films (Figure S8) 8

3-Dimensional inspection of photo-Fabricated P3HCA film (Figure S9) 9

Results of the photofabrication experiments (Table S1 and S2) 10 
a)

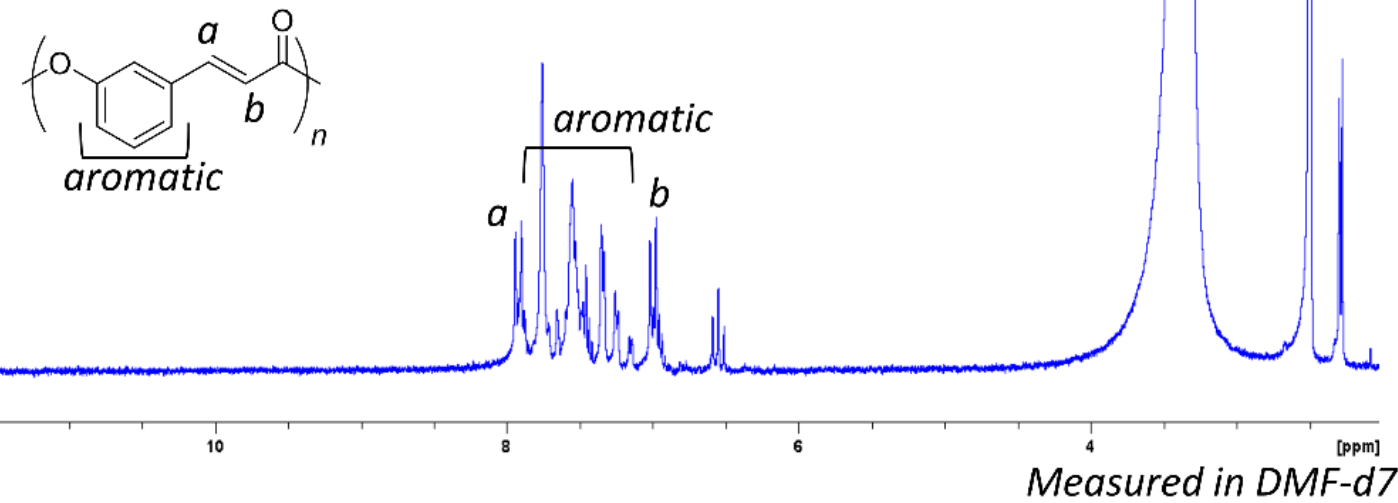

b)

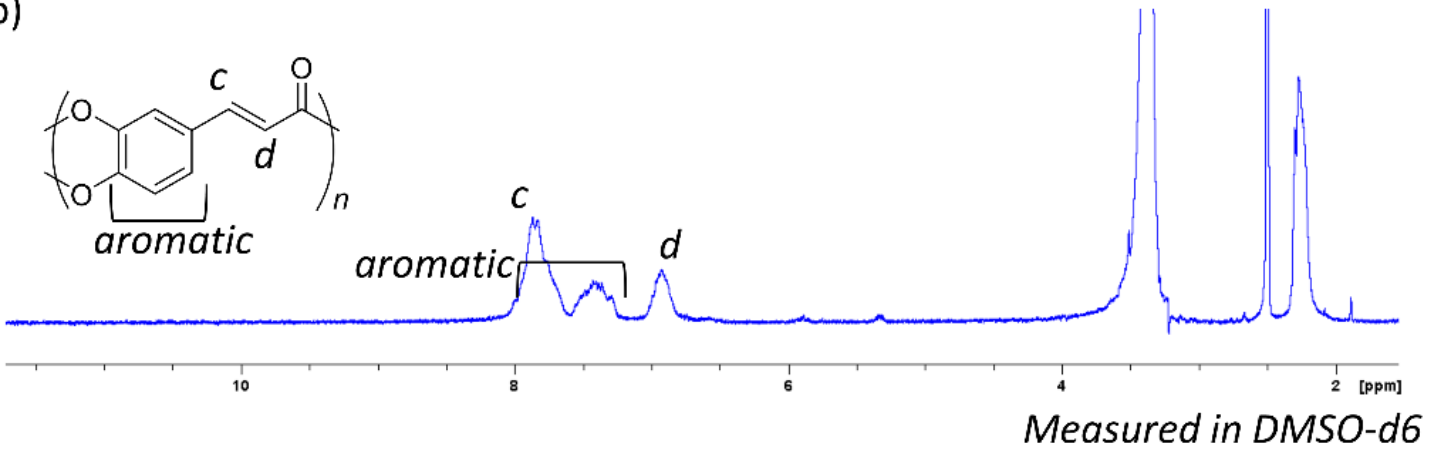

Figure S1. ${ }^{1} \mathrm{H}$ NMR spectra of the obtained (a) P3HCA and (b) PdHCA (400 MHz). 


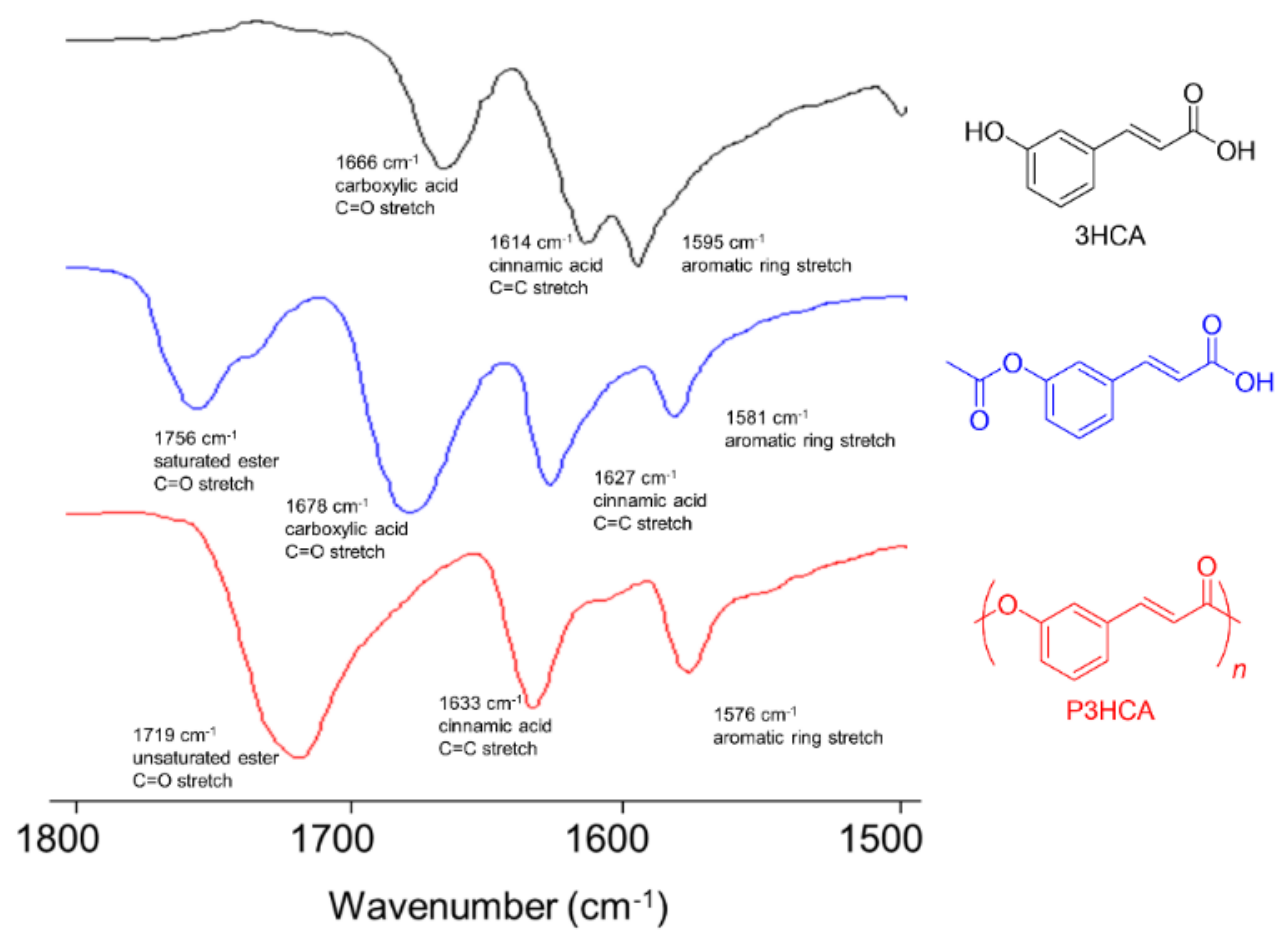

Figure S2. FT-IR spectra of the obtained 3HCA, acetylated 3HCA, and P3HCA.

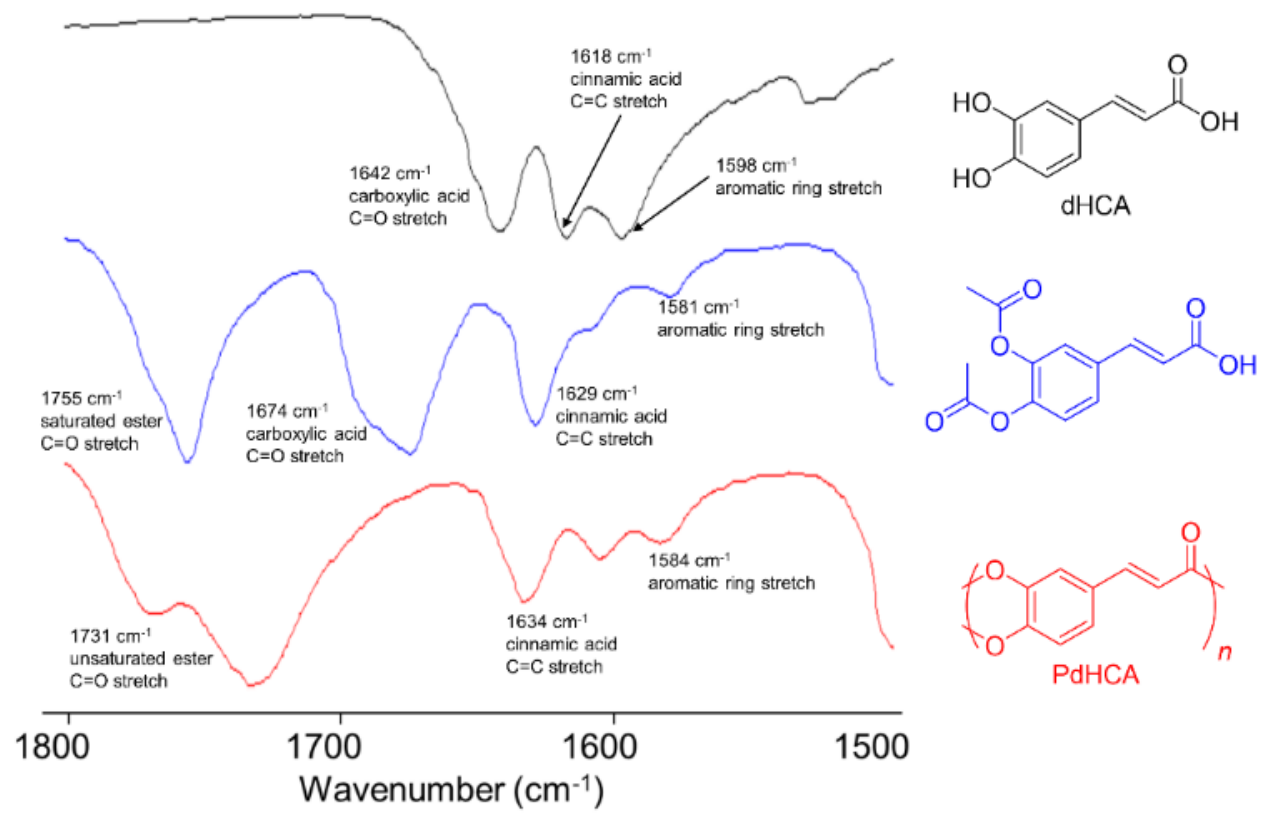

Figure S3. FT-IR spectra of the obtained dHCA, acetylated dHCA, and PdHCA. 

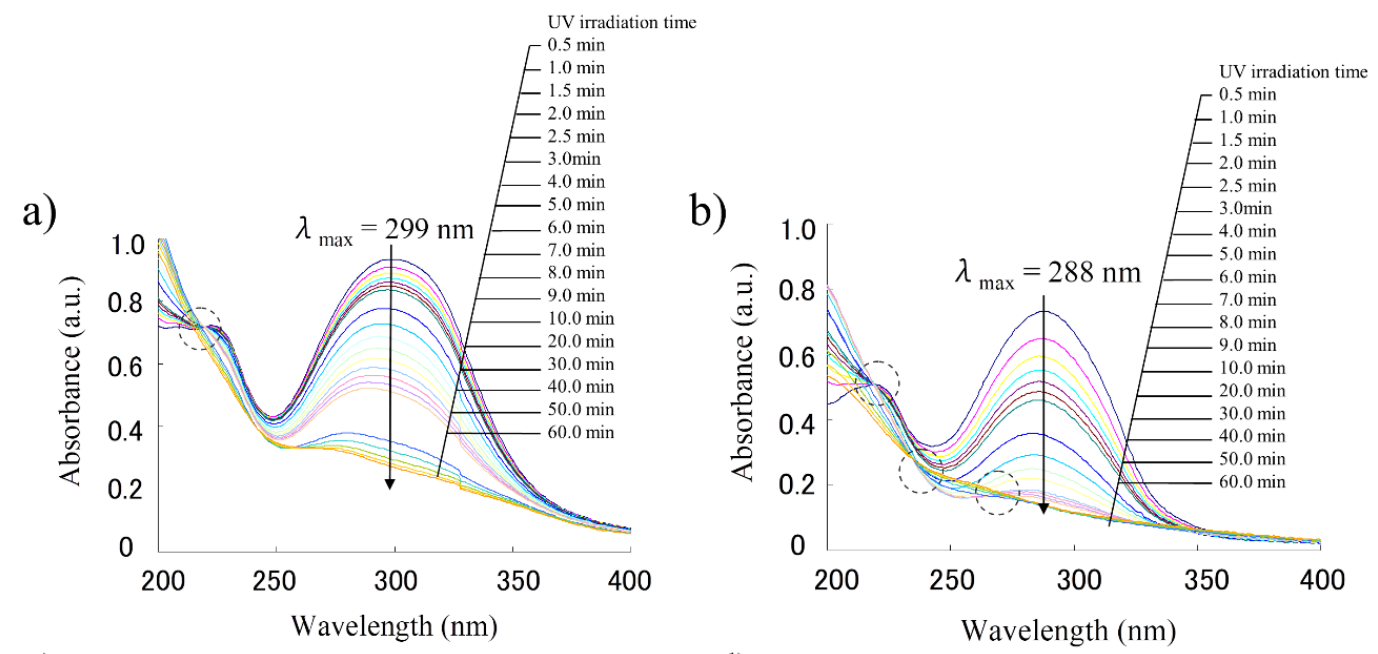

c)

d)
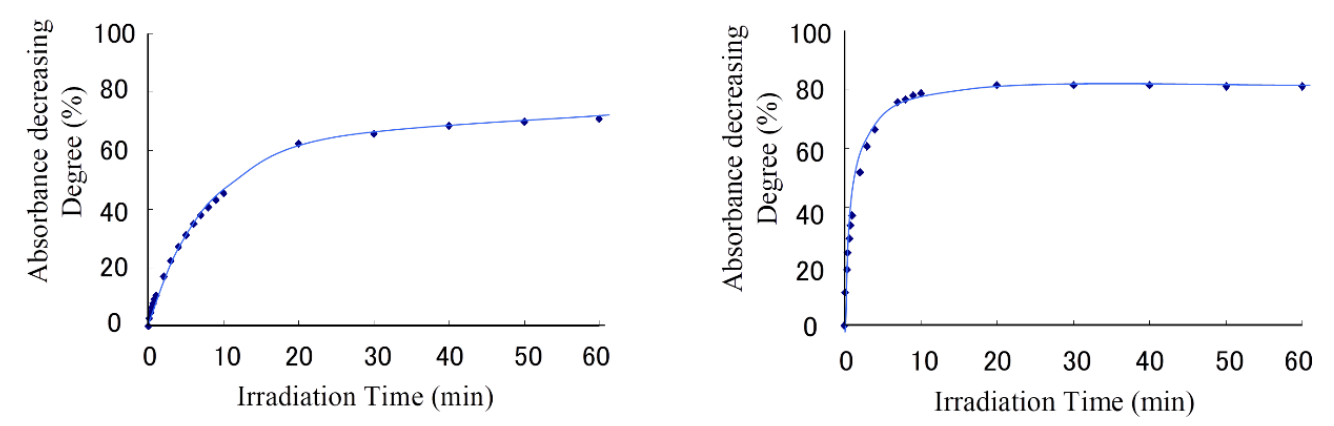

Figure S4. Changes in UV-vis spectra of (a) PdHCA and (b) P3HCA. Crosslinking degrees of (c) PdHCA and (d) P3HCA films, measured thin film on quartz plate as a function of UV irradiation time.

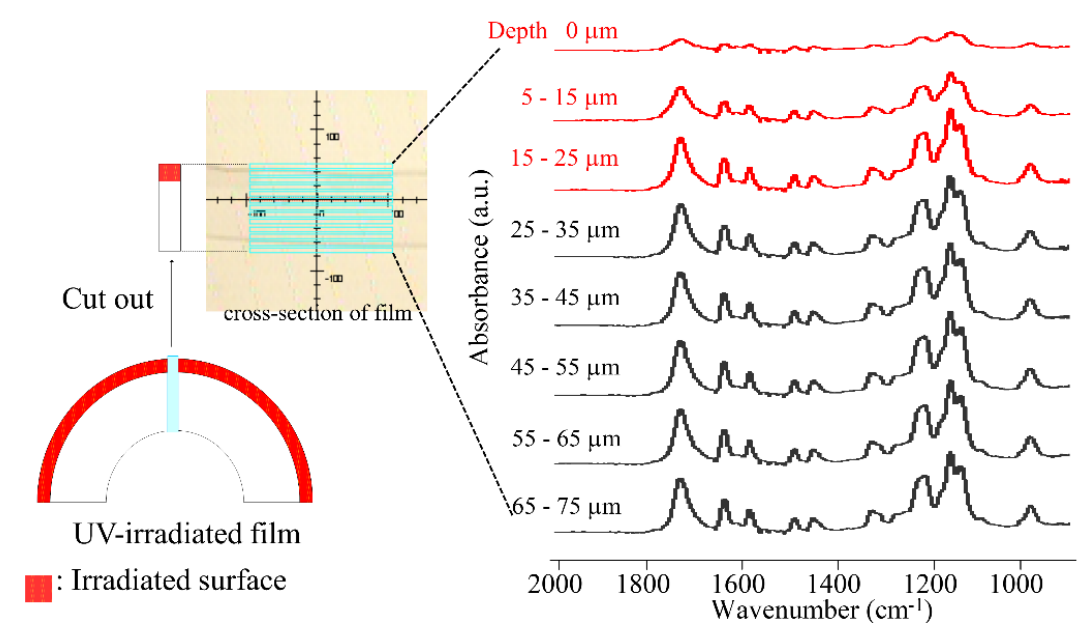

Figure S5. IR/ATR spectra of cross-section of P3HCA film. The peaks of vinylene were disappear at 1640 and $980 \mathrm{~cm}^{-1}$ in $0-25 \mu \mathrm{m}$ depth from film surface. 
a)

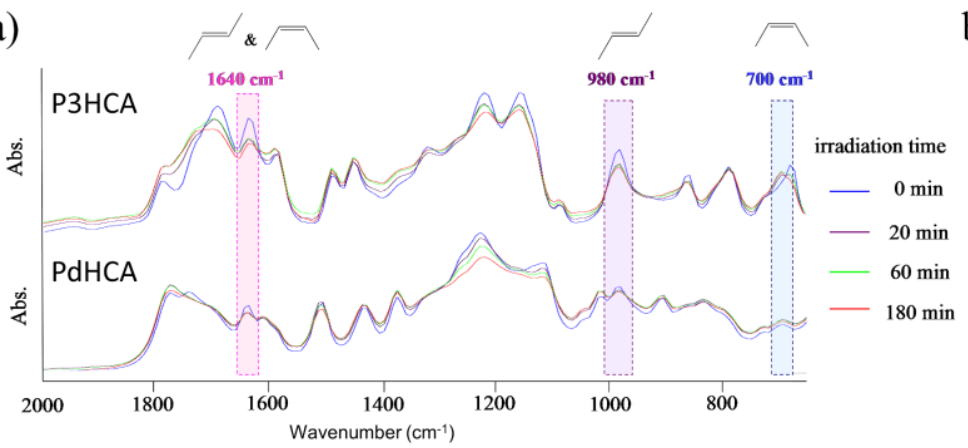

b)

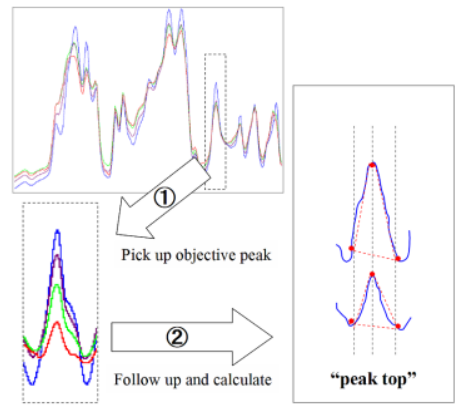

Figure S6. (a) Extracted IR-RA spectra of P3HCA and PdHCA film from step scanning during UV irradiation. (b) The process of derived peak top from original absorbance of IR spectra.

The continuous IR step scanning result during UV irradiated at 0, 20,60 and 180 min were extracted for checking the changes of the peaks such as $-\mathrm{CH}=\mathrm{CH}-$ part at 1640,980 and $700 \mathrm{~cm}^{-1}$. The peak of $980 \mathrm{~cm}^{-1}$ was assigned to trans style vinyl bond, and the peak of $700 \mathrm{~cm}^{-1}$ was assigned to cis style vinyl bond. Furthermore, the peak of $1640 \mathrm{~cm}^{-1}$ contained both style of vinyl bond. The objective peaks were calculated its peak top absorbance from original IR spectra, and drawn to time-resolved IR absorbance change. 

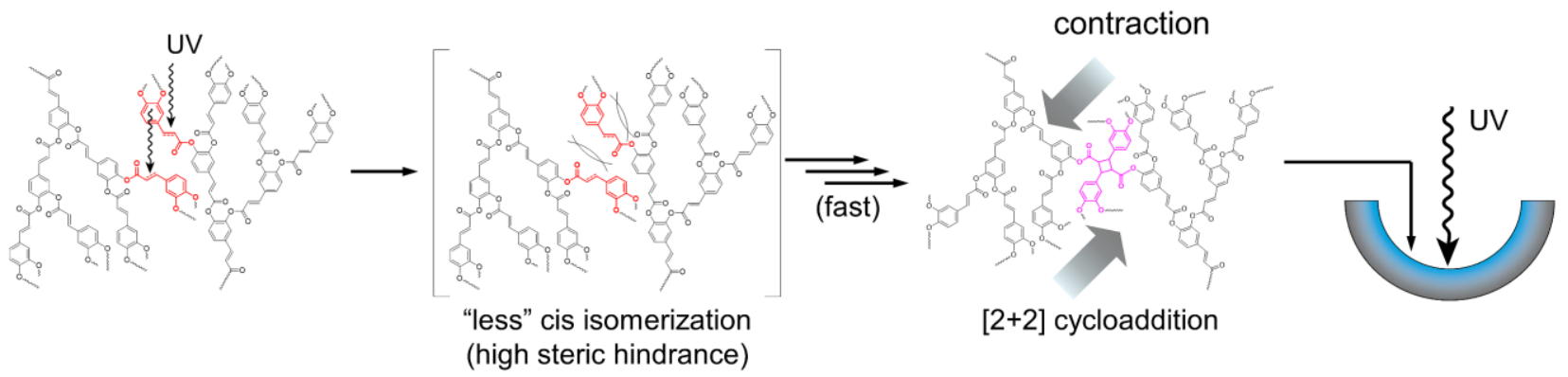

Figure S7. Schematic illustration of structural changes for PdHCA; photocontraction by UV irradiation: Also in PdHCA, it passes through the excited state by UV irradiation, but cis isomerization does not occur due to the large steric hindrance between the each molecular chains, and $[2+2]$ cycloaddition was prioritized. As a result, the film exhibits contraction behavior. 

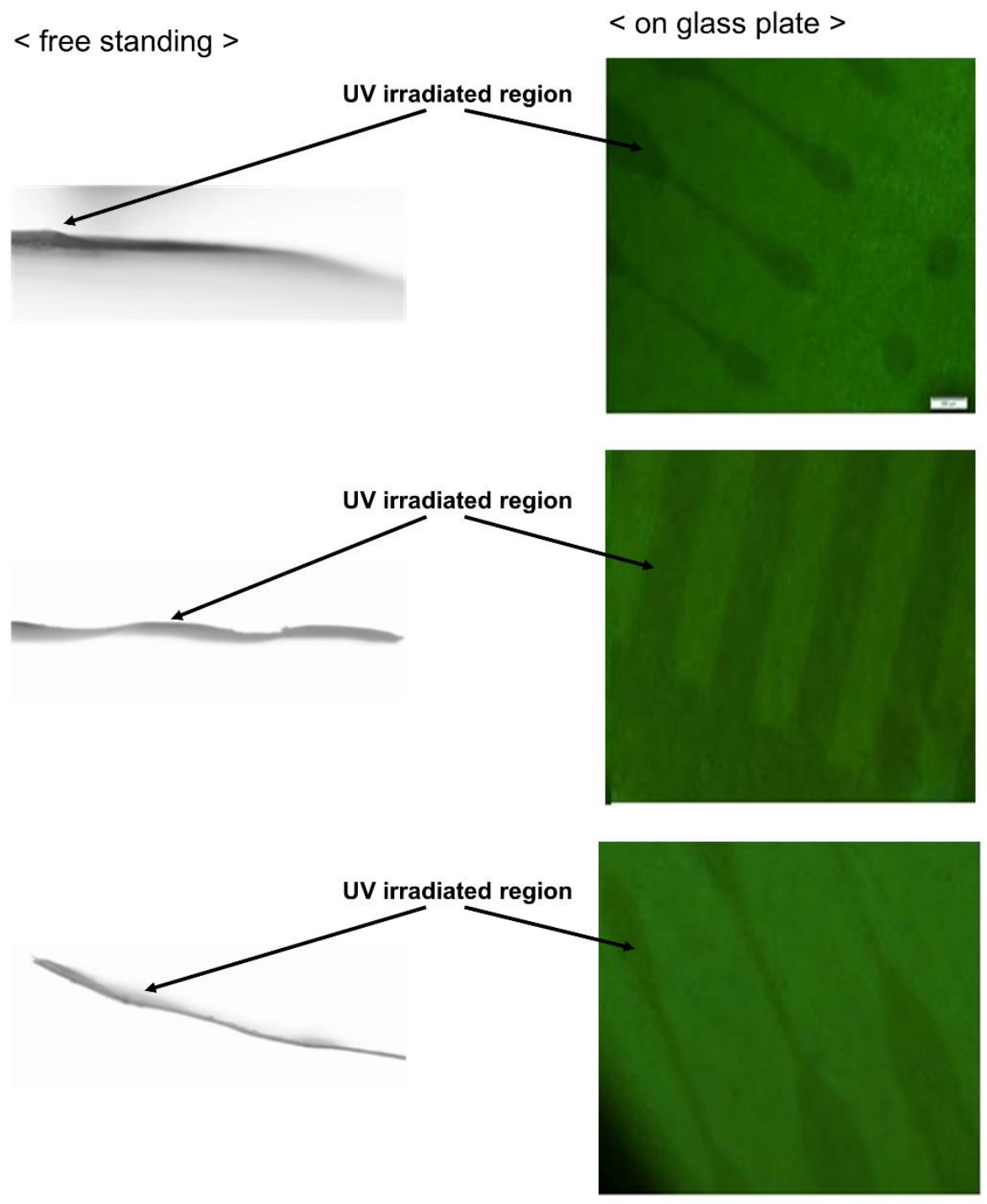

Figure S8. Different deformation pattern of P3HCA films with the free-standing and on glass plate. 

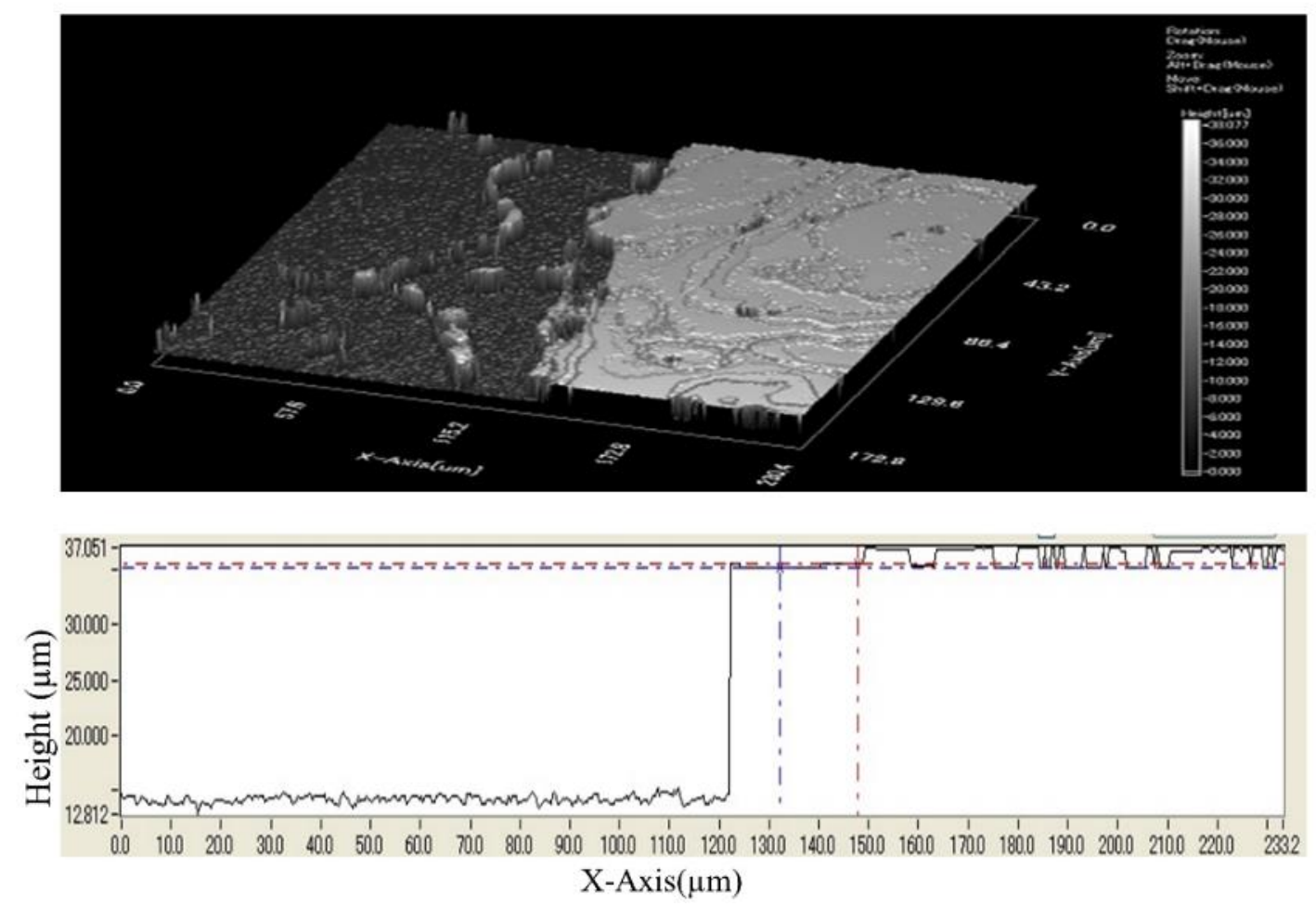

Figure S9. 3-Dimensional inspection of photo-fabricated P3HCA film on glass plate. 
Table S1. Dependence of the bending angle of the P3HCA film without glass plate on UV-irradiation time

\begin{tabular}{|c|c|c|c|c|}
\hline S No. & $\begin{array}{c}\text { Distance }^{a} \\
(\mathrm{~cm})\end{array}$ & Time & $\begin{array}{l}\text { UV Intensity } \\
\left(\mathrm{mW} / \mathrm{cm}^{2}\right)\end{array}$ & Bending Angle \\
\hline $\mathrm{S} 1$ & - & 0 & - & 0 \\
\hline $\mathrm{S} 2$ & 4 & 5 & 46.28 & $12.3 \pm 0.45$ \\
\hline S3 & 4 & 10 & 47.37 & $20.3 \pm 2.26$ \\
\hline $\mathrm{S} 4$ & 4 & 15 & 43.61 & $22.7 \pm 2.23$ \\
\hline S5 & 4 & 20 & 45.23 & $25.5 \pm 3.10$ \\
\hline S6 & 4 & 30 & 48.16 & $25.9 \pm 1.90$ \\
\hline
\end{tabular}

${ }^{a}$ Distance of sample and UV light source. ${ }^{b}$ Observed from the angle of the non-UV-irradiated surface (flat) and the deformed portion generated on the irradiated surface. 
Table S2. Dependence of the bending angle of the P3HCA film with glass plate on UV-irradiation time

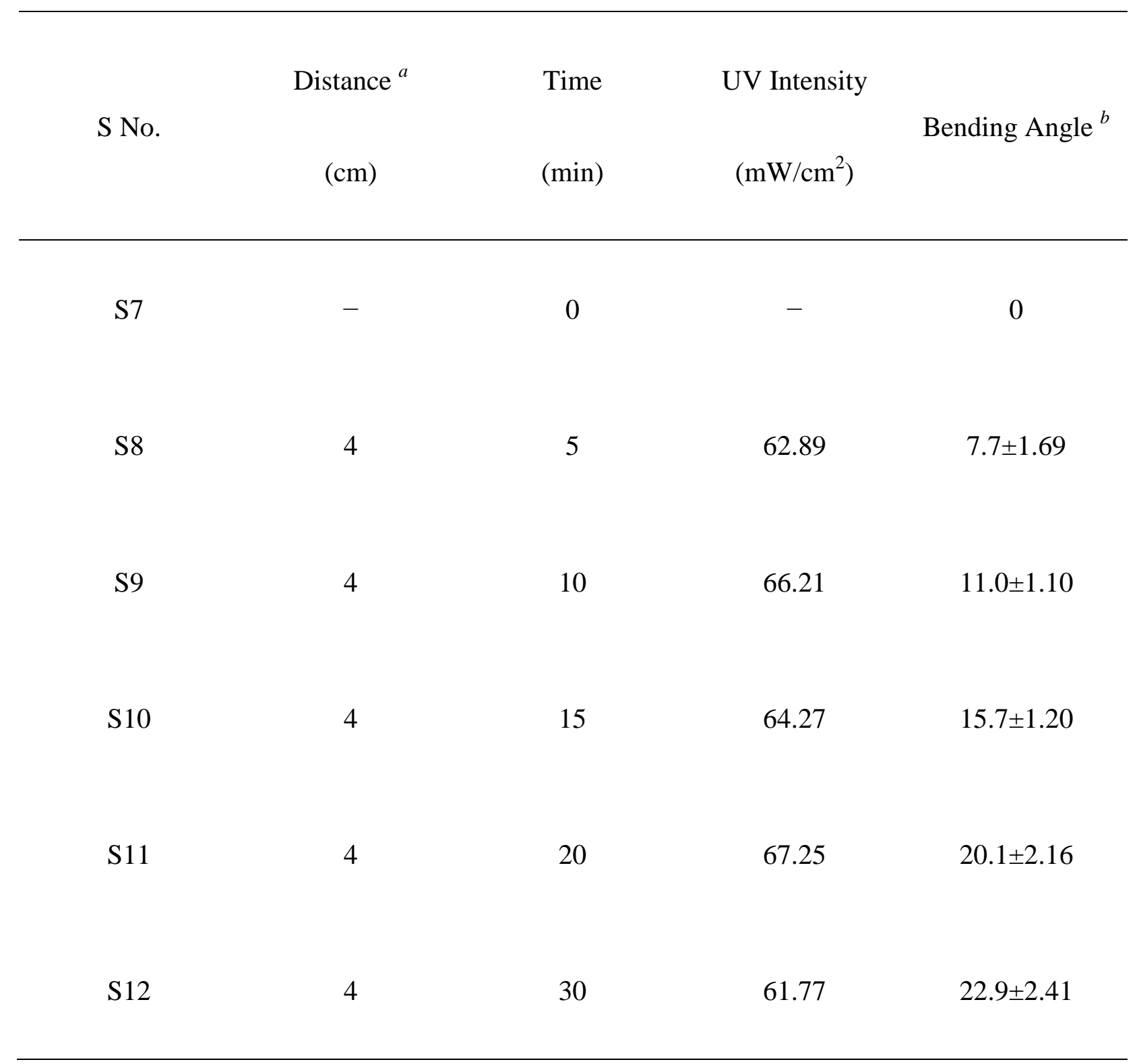

${ }^{a}$ Distance of sample and UV light source. ${ }^{b}$ Observed from the angle of the non-UV-irradiated surface (flat) and the deformed portion generated on the irradiated surface. 